\title{
Regulation of RIP3 protein stability by PELI1-mediated proteasome-dependent degradation
}

\author{
Han-Hee Park ${ }^{1,4}$, Michael J. Morgan ${ }^{2}$, Ho Chul Kang ${ }^{3,4}$ \& You-Sun Kim ${ }^{1,4, *}$ \\ ${ }^{1}$ Department of Biochemistry, Ajou University, School of Medicine, ${ }^{2}$ Department of Natural Sciences, Northeastern State University, \\ Oklahoma 74464, USA, ${ }^{3}$ Department of Physiology, Ajou University, School of Medicine, ${ }^{4}$ Department of Biomedical Sciences, Graduate \\ School, Ajou University, Suwon 16499, Korea
}

Receptor-interacting protein kinase-3 (RIP3 or RIPK3) is a serine-threonine kinase largely essential for necroptotic cell death; it also plays a role in some inflammatory diseases. High levels of RIP3 are likely sufficient to activate necroptotic and inflammatory pathways downstream of RIP3 in the absence of an upstream stimulus. For example, we have previously detected high levels or RIP3 in the skin of Toxic Epidermal Necrolysis patients; this correlates with increased phosphorylation of MLKL found in these patients. We have long surmised that there are molecular mechanisms to prevent anomalous activity of the RIP3 protein, and so prevent undesirable cell death and inflammatory effects when inappropriately activated. Recent discovery that Carboxyl terminus of Hsp 70-Interacting Protein (CHIP) could mediate ubiquitylation- and lysosomedependent RIP3 degradation provides a potential protein that has this capacity. However, while screening for RIP3-binding proteins, we discovered that pellino E3 ubiquitin protein ligase 1 (PELI1) also interacts directly with RIP3 protein; further investigation in this study revealed that PELI1 also targets RIP3 for proteasome-dependent degradation. Interestingly, unlike CHIP, which targets RIP3 more generally, PELI1 preferentially targets kinase active RIP3 that has been phosphorylated on T182, subsequently leading to RIP3 degradation. [BMB Reports: Perspective 2018; 51(10): 484-485]

*Corresponding author. E-mail: yousunkim@ajou.ac.kr

https://doi.org/10.5483/BMBRep.2018.51.10.217

Received 3 September 2018

Keywords: Necroptosis, PELI1, Proteasome, RIP3, TEN

Abbreviations: CHIP, carboxyl terminus of Hsp 70-interacting protein; FHA, forkhead associated; PELI1, pellino E3 ubiquitin protein ligase 1; RIP3, receptor interacting protein 3; TEN, toxic epidermal necrolysis

Perspective to: Seung-Won Choi and Han-Hee Park et al. (2018) PELI1 Selectively Targets Kinase-Active RIP3 for UbiquitylationDependent Proteasomal Degradation, Molecular Cell, 70, 920-935, https://doi.org/10.1016/j.molcel.2018.05.016
Necroptosis is a form of regulated cell death morphologically and biochemically distinct from apoptosis. Primarily, this means that, unlike apoptosis, necroptosis leads to high membrane permeability and loss of cellular contents, many of which can lead to immunogenic responses. At the molecular level, necroptosis critically depends on activation of receptor-interacting protein kinase3 (RIP3 or RIPK3). As a key regulator of necroptosis, RIP3 expression has been altered in several human pathological conditions (Grootjans $\mathrm{S}$ et al. (2017) Cell Death Differ 24, 1184-1195). Previously, we and other groups have demonstrated that RIPK3 expression is significantly reduced or lost in various cancer cell lines and cancer tissues, leading to resistance to necroptosis in these cells; extensive loss/reduction of RIPK3 expression suggests its loss provides some kind of selective advantage to tumor cells (Koo GB et al. (2015) Cell Res 25, 707-725). In contrast with cancer, high levels of RIP3 expression have been detected in inflammatory bowel disease, chronic obstructive pulmonary disease, multiple sclerosis, and, as we have recently reported, toxic epidermal necrolysis (Kim SK et al. (2015) J Invest Dermatol 135, 2021-2030). Thus, there are multiple studies that suggest that regulation of RIP3 expression has multiple therapeutic implications.

Ubiquitin-mediated signaling is involved in regulating the activity and/or stability of many TNF-induced necroptosis pathway components, so it is unsurprising that ubiquitylation of RIP3 would be highly important for its regulation, and RIP3 ubiquitylation has been often reported on different RIP3 residues, though consequences of such are being sorted out. Deubiquitylating enzyme A20 can reverse K5 RIP3 ubiquitylation, which promotes necrosome assembly and necroptosis (Onizawa M et al. (2015) Nat Immunol 16, 618627). So far only Carboxyl terminus of Hsp 70-interacting protein (CHIP) has been revealed to mediate ubiquitylationassociated RIP3 degradation (though this is lysosomedependent, and not proteasome dependent), consequently leading to regulation of necroptosis (Seo J et al. (2016) Nat Cell Biol 18, 291-302).

In our recent study, our screen of proteome microarrays revealed that the pellino E3 ubiquitin protein ligase 1 (PELI1) is a novel direct binding partner of RIP3. We further demonstrate 
that PELI1 targets RIP3 protein for proteasome-dependent degradation (Choi SW et al. (2018) Mol Cell 70, 920-935). This is the first report of a E3 ligase-directed ubiquitylation event that leads to proteasome-dependent degradation of RIP3. We identified RIP3 K363 as the main target for PELI1-mediated ubiquitylation by mass spectrometric analysis; this is one of several RIP3 lysines (K55, K89, K363, K501) reported to be modified by CHIP. However, only K363R mutation abolishes ubiquitylation by PELI1 and prevents PELI1-mediated degradation, consistent with our mass spectrometric data. Though we observed CHIP-dependent ubiquitylation and degradation in overexpression assays, in our hands we were unable to observe upregulation of endogenous RIP3 expression in response to lysosomal inhibition. Treatment of cells with proteasome inhibitors, however, led to increased levels of RIP3 protein. Additionally, PELI1-facilitated RIP3 degradation was only prevented by proteasome inhibitors (MG132 and BTZ) but not by lysosome inhibitors (CQ and E64d/Pep A) indicating that PELI1 controls RIP3 protein destabilization via ubiquitylation-dependent proteasome-mediated degradation.

One of our more interesting finding was that, unlike CHIP, which targets RIP3 protein generally, PELI1 associates with,

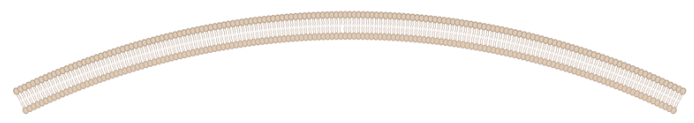

CHIP-RIP3 flux (phospho-independent lysosomal degradation)

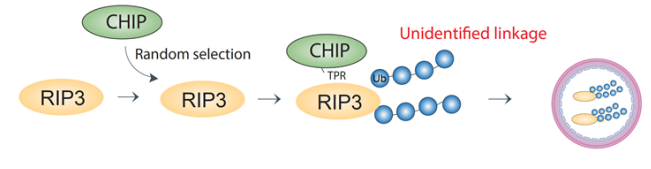

PELI1-RIP3 flux (phospho-dependent proteosomal degradation)

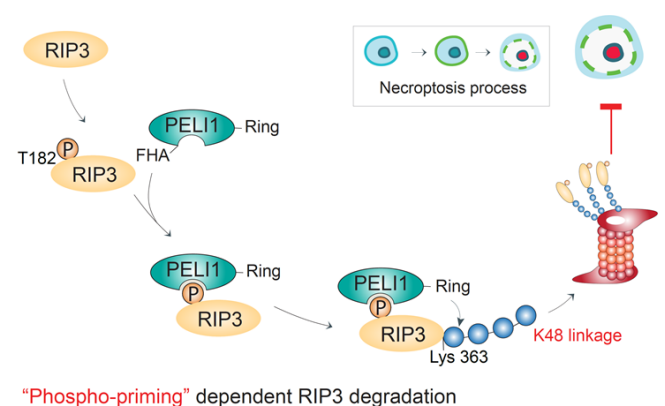

Diagram 1. Schematic diagram of a novel, proteasome-dependent mode of RIP3 degradation mediated by an E3 ubiquitin ligase, PELI1. Phosphorylation of RIP3 on T182 leads to interaction with the FHA domain of PELI1 and PELI1-mediated K48-linked polyubiquitylation of RIP3 at K363 leads to its proteasome-dependent degradation. Regulation of activated RIP3 by PELI1 provides a homeostatic mechanism to prevent aberrant cell death and minimize necroptotic pathology. and then preferentially target, kinase-active RIP3. We showed, for example, that kinase inactive RIP3 was neither ubiquitylated nor degraded by PELI1. Further investigation revealed that phosphorylation of RIP3 on T182, which only occurs in kinase-active RIP3 (presumably by autophosporylation) leads to interaction with the FHA (forkhead associated) domain of PELI1. Thus, kinase-inactive RIP3 fails to interact with PELI1, which explains why there is lack of ubiquitylation. Disruption of a specific T182 within the active loop of RIP3 blocks recognition by PELI1 and also prevents RIP3 ubiquitylation and degradation. Though ubiquitylation of T182A RIP3 by PELI1 was substantially reduced, the T182A mutant was capable of being ubiquitylated by $\mathrm{CHIP}$, and this led to its degradation. Thus, though both CHIP and PELI1 interact with and ubiquitylate RIP3, there is a substantial difference in how they recognize RIP3. We hypothesize that this may allow for fine tuning of basal levels of necroptotic signaling within a cell, with CHIP regulating RIP3 on a general level, and PELI1 functioning within a signaling complex to control the strength of a necroptotic response. Interestingly, we showed that phospho-T182 could function as a priming site for the auto-phosphorylation of Ser-227. That is, phospho-T182 is required for phosphorylation of S227, a phosphorylation event that has long been used as a marker for RIP3 activation. This may indicate that T182 is a crucial amino acid for RIP3 kinase activity (Diagram 1).

High RIP3 expression has been reported to lead to spontaneous auto-phosphorylation and inopportune necroptosis; we have hypothesized that cells may need a compensation mechanism(s) to prevent such occurrences. Thus, PELI1mediated degradation of kinase active RIP3 may represent one such mechanism to prevent aberrant RIP3 activation. We previously reported elevated RIP3 expression in Toxic Epidermal Necrolysis (TEN), a life-threatening dermatological inflammatory condition; this high expression is correlated with MLKL phosphorylation. In this study, we found that RIP3 was negatively correlated with PELI1 expression when examining skin from normal and TEN patients. Our data reveal that low expression of PELI1 results in high expression of RIP3 in keratinocytes; low levels of PELI1 in patients may contribute to necroptotic cell death in TEN patients via high RIP3 levels and subsequent MLKL activation. Our findings suggest that maintaining PELI1 expression in keratinocytes would help to prevent keratinocyte death and may be a potential therapeutic target for TEN treatment.

\section{ACKNOWLEDGEMENTS}

This study was supported by the National Research Foundation of Korea (NRF) grant funded by the Korea government (No. 2017R1A2B3002343) and grant of the Korea Health Technology R\&D Project through the Korea Health Industry Development Institute (KHIDI), funded by the Ministry of Health \& Welfare, Republic of Korea (No. HI15C0554). 